\title{
Effect of Candida albicans bronchial colonization on hospital- acquired bacterial pneumonia in patients with systemic lupus erythematosus
}

\author{
Yuetian Yu ${ }^{1,2 \#}, \mathrm{Jia} \mathrm{Li}^{1 \#}$, Suli Wang ${ }^{1}$, Yuan $\mathrm{Gao}^{2}$, Hui Shen ${ }^{3}$, Liangjing Lu' \\ ${ }^{1}$ Department of Rheumatology, ${ }^{2}$ Department of Critical Care Medicine, Ren Ji Hospital, School of Medicine, Shanghai Jiao Tong University, Shanghai \\ 200001, China; ${ }^{3}$ Department of Laboratory Medicine, Shanghai East Hospital, Tongji University School of Medicine, Shanghai 200123, China \\ Contributions: (I) Conception and design: YT Yu; (II) Administrative support: LJ Lu; (III) Provision of study materials or patients: J Li and H Shen; \\ (IV) Collection and assembly of data: All authors; (V) Data analysis and interpretation: SL Wang; (VI) Manuscript writing: All authors; (VII) Final \\ approval of manuscript: All authors. \\ "These authors contributed equally to this work. \\ Correspondence to: Hui Shen. Department of Laboratory Medicine, Shanghai East Hospital, Tongji University School of Medicine, Shanghai 200123, \\ China. Email: shenhui0712@163.com; Liangjing Lu. Department of Rheumatology, Ren Ji Hospital, School of Medicine, Shanghai Jiao Tong \\ University, Shanghai 200001, China. Email: lu_liangjing@163.com.
}

Background: To investigate the effect of Candida albicans airway colonization on hospital-acquired bacterial pneumonia (HABP) in patients with systemic lupus erythematosus (SLE).

Methods: SLE patients with HAP were enrolled in this retrospective cohort study from January 2015 to December 2018. According to the culture results of respiratory tract secretions and antifungal treatment, patients were divided into non-C. albicans colonization group (NCG), C. albicans colonization with antifungal treatment group (CTG) and C. albicans colonization without antifungal treatment group (CNTG). Twentyeight days of all-cause mortality after the onset of HABP of each group was assessed, total duration of antibiotic treatment, changes of inflammatory cytokines and markers of immune status were documented.

Results: A total of 269 SLE patients with HABP were finally included in the study. Among them, 69.1\% (186/269) were found C. albicans airway colonization, and 36.1\% (97/269) received antifungal treatment. No difference of 28 days of all-cause mortality after HABP onset was found among the three groups $(10.8 \%$ vs. $13.4 \%$ vs. $15.7 \%, \mathrm{P}>0.05)$. Duration of antibiotic treatment of HABP due to Acinetobacter baumannii was shorter in CTG $(11.21 \pm 2.45$ days $)$ than that in CNTG $(12.89 \pm 3.87$ days $)$ as well as HABP due to Pseudomonas aeruginosa $(10.57 \pm 2.66$ vs. $12.56 \pm 3.04$ days $)(\mathrm{P}<0.05$, respectively). Antifungal treatment could decrease the level of interleukin-1 $\beta$, interleukin-6, tumor necrosis factor- $\alpha$ and interferon- $\gamma$ in CTG than in CNTG ( $\mathrm{P}<0.05$, respectively). In addition, it could regulate immune function by decrease the concentration of interleukin-17 and elevate the counts of CD4+Th lymphocyte as well ( $\mathrm{P}<0.05$, respectively).

Conclusions: $C$. albicans airway colonization was not associated with HABP mortality in SLE patients. Antifungal therapy in these patients might shorten the duration of antibiotics in treating HABP due to $A$. baumannii and P. aeruginosa.

Keywords: Candida albicans; colonization; systemic lupus erythematosus (SLE); hospital-acquired pneumonia; immunocompromised host (ICH)

Submitted Sep 07, 2019. Accepted for publication Sep 25, 2019.

doi: $10.21037 /$ atm.2019.10.44

View this article at: http://dx.doi.org/10.21037/atm.2019.10.44 


\section{Introduction}

Isolation of Candida albicans from respiratory secretions is not uncommon in immunocompromised hosts (ICHs) especially in those with systemic lupus erythematosus (SLE) due to immunosuppressive agents and high doses of glucocorticoid treatment (1). According to the practice guideline, it is indicative of colonization, and antifungal therapy is rarely necessary (2). However, the co-existence of bacteria and fungi has aroused great concern in recent years. Based on some in vitro studies, there is growing convincing evidence shows that interplay of fungi and bacteria does exist through quorum-sensing (QS) molecules, but its clinical significance is hard to evaluate in clinical practice $(3,4)$. Although some studies indicated that airway Candida spp. colonization might increase the incidence of bacterial pneumonia, while others came to the opposite conclusion (5-7).

What is more, does empirical antifungal therapy could improve the prognosis of hospital-acquired bacterial pneumonia (HABP) in ICHs with $C$. albicans airway colonization is still on debate (7-9). Thus, our retrospective study was conducted to clarify the status of the following objectives: (I) does effect of $C$. albicans airway colonization on HABP really exist in patients with SLE and (II) does empirical antifungal treatment might improve the prognosis of these patients.

\section{Methods}

\section{Research brief}

This retrospective cohort study was performed in Ren Ji hospital. Totally 90 inpatient beds were occupied by the department of rheumatology and critical care medicine. Ethics Committee was approved by Shanghai Jiao Tong University (No. 2016-Clinical-Res-011).

\section{Study population}

Patients with SLE who were clinically diagnosed hospitalacquired pneumonia (HAP) were enrolled in the study from January 2015 to December 2018. Patients were excluded if (I) extrapulmonary infection was found when they admitted to hospital or during their hospital stay; (II) pathogen detection results were lacked or negative; (III) non-albicans Candida (NAC) species was isolated; (IV) they were moribund or not expected to survive $48 \mathrm{~h}$ because of an underlying irreversible medical condition after the onset of HABP and (V) had an incomplete medical history. According to the culture results of respiratory tract secretions and antifungal treatment, patients were divided into non-C. albicans colonization group (NCG), C. albicans colonization with antifungal treatment group (CTG) and $C$. albicans colonization without antifungal treatment group (CNTG).

\section{Diseases definition}

Patients that fulfilled the definition criteria of British Society for Rheumatology (BSR) were diagnosed SLE (10), and clinical practice guidelines by American Thoracic Society (ATS) and Infectious Diseases Society of America (IDSA) were applied to diagnose HABP which was described as follows: (I) pneumonia acquired $48 \mathrm{~h}$ after hospital admission; (II) new pulmonary infiltrate detected on chest radiograph; (III) pathogens were detected from the respiratory tract secretions and (IV) with at least two of the following criteria: (i) an abnormal temperature ( $<35.6$ or $>37.8^{\circ} \mathrm{C}$ ); (ii) leucocytosis $\geq 10 \times 10^{9} / \mathrm{L}$ or $\leq 4 \times 10^{9} / \mathrm{L}$ and (iii) newly purulent sputum (11).

\section{Strains identification and antimicrobial susceptibility testing}

Pathogens that caused HABP and their antimicrobial susceptibility results were obtained from Ren Ji hospital's microbiological chamber database. Strains were identified using a VITEK-2 automated system (bioMérieux), and antimicrobial susceptibility testing was determined according to the standards provided by the Clinical and Laboratory Standards Institute (CLSI) (http://ncipd. org/control/images/NCIPD_docs/CLSI M100-S28. pdf). Multidrug-resistant (MDR) was defined as acquired non-susceptibility to at least one agent in three or more antimicrobial categories (12). Only the first isolated strain was tested in our study.

\section{Data collection and clinical assessment}

Information on SLE patients with HABP was obtained from the hospital's electronic medical records system. The demographic and clinical characteristics of each patient enrolled comprised seven parts: (I) basic information including age, gender, and comorbidity; (II) preadministration antibiotics and antifungal agent before hospital admission; (III) therapeutic schemes of SLE 


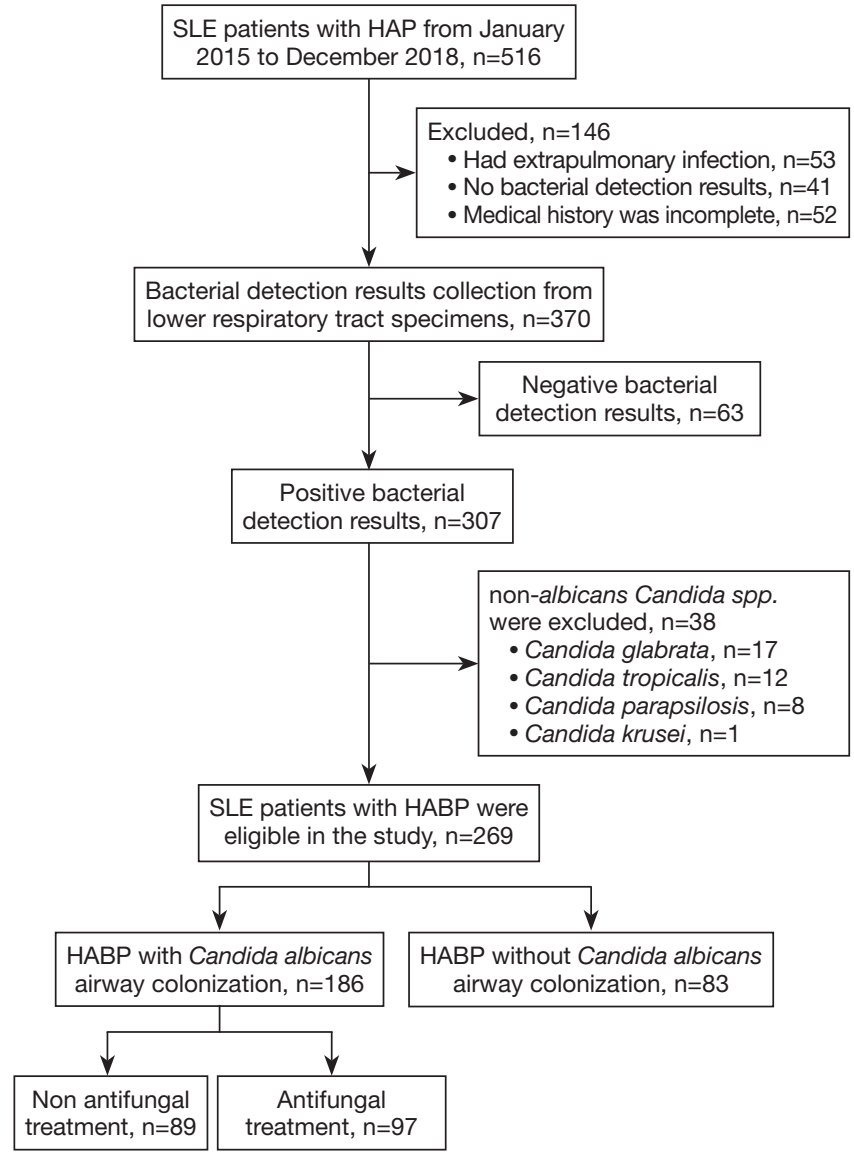

Figure 1 Flow chart of the study. Patients with HABP enrolled in the study and the distribution of the Candida albicans. HAP, hospital-acquired pneumonia; HABP, hospital-acquired bacterial pneumonia; SLE, systemic lupus erythematosus.

including the administration of immunosuppressant, daily prednisone dose and disease activity; (IV) duration of antibiotics treatment; (V) changes of inflammation cytokines like procalcitonin (PCT), C-reactive protein (CRP), interleukin-6 (IL-6), IL-1 $\beta$, interferon- $\gamma(\mathrm{INF}-\gamma)$ and tumor necrosis factor- $\alpha$ (TNF- $\alpha$ ); (VI) variation tendency of immune parameters like IgA, IL-17 and the count of CD4+Th lymphocyte [markers included in (V) and (VI) were tested at the time of admission and weekly according to the procedure of HABP treatment in our department as well as immune parameters)], and (VII) outcomes of the patients which included 28 days of all-cause mortality after the onset of HABP and the rate of invasive or non-invasive mechanical ventilation.

Duration of antibiotic therapy was defined as antibiotic administration for treating HABP during the hospitalization, which was based on the time to clinical stability (TCS). According to the guidelines of National Institute for Health and Care Excellence (NICE) (13), the criteria for clinical stability were defined as follows: (I) lack of fever for $>8 \mathrm{~h}$; (II) improvement in clinical signs (cough and shortness of breath); (III) improvement in leucocytosis (decrease $>10 \%$ from the previous day); and (IV) tolerance of oral antibiotic intake. The criteria for TCS was evaluated daily by attending physicians (14).

\section{Outcomes of the study}

We primarily assessed the all-cause mortality of HABP over a 28-day treatment. In addition, the total duration of antibiotic treatment, evolution of inflammation cytokines and immune parameters were also compared.

\section{Statistical analysis}

Statistical analysis was performed by using SPSS version 22.0 (IBM for windows). Characteristics among the three groups were compared using the chi-square test for caegorical variables and using analysis of variance or Kruskal-Wallis test for continuous variables. Bonferroni adjustments were used for multiple comparisons. Variables between the C. albicans colonization with or without antifungal treatment group were compared by Student's t-test and were expressed as the means (standard deviations), while nonnormally distributed data were compared by the Wilcoxon rank-sum test and reported as medians (interquartile range). Factors associated with C. albicans airway colonization and MDR pathogens isolation were identified by multivariate logistic regression and summarized with odds ratios (ORs) and 95\% confidence intervals (CIs). A two-sided $\mathrm{P}<0.05$ was considered statistically significant. Figures were drawn by GraphPad Prism version 7.0.

\section{Results}

\section{Clinical features of the study population}

Totally 516 SLE patients with HAP were screened in the medical record system, and 269 with HABP were finally enrolled in the study. Among them, 69.1\% (186/269) were found C. albicans airway colonization, and 36.1\% (97/269) received antifungal treatment (Figure 1).

Age, gender, course of SLE, and systemic lupus erythematosus disease activity index (SLEDAI) did not 
Table 1 Demographics and clinical characteristics of SLE patients with HABP

\begin{tabular}{|c|c|c|c|c|c|c|}
\hline Characteristics & NCG $(n=83)$ & CTG $(n=97)$ & CNTG $(n=89)$ & $P$ value ${ }^{a}$ & $P$ value ${ }^{b}$ & $P$ value ${ }^{c}$ \\
\hline Gender, female, n (\%) & 79 (95.2) & $96(98.9)$ & $87(97.7)$ & 0.273 & 0.266 & 0.941 \\
\hline Course of SLE, month (mean \pm SD) & $38.9 \pm 8.4$ & $38.3 \pm 9.2$ & $40.2 \pm 7.3$ & 0.407 & 0.589 & 0.123 \\
\hline \multicolumn{7}{|l|}{ SLE activity at the time of infection, $\mathrm{n}(\%)$} \\
\hline Hematological activity & $5(6.1)$ & $7(7.2)$ & $7(7.9)$ & 0.893 & 0.657 & 0.867 \\
\hline Central nervous system activity & $3(3.6)$ & $4(4.1)$ & $3(3.4)$ & 0.962 & 0.772 & 0.908 \\
\hline SLEDAI score $($ mean \pm SD) & $9.3 \pm 2.7$ & $8.7 \pm 3.1$ & $8.6 \pm 4.2$ & 0.271 & 0.143 & 0.853 \\
\hline Immunosuppressive therapy during hospitalization, n (\%) & $11(13.3)$ & $24(24.7)$ & $25(28.1)$ & 0.05 & 0.017 & 0.605 \\
\hline $\begin{array}{l}\text { Broad-spectrum antibiotic prescription within } 30 \text { days before } \\
\text { admission, } \mathrm{n}(\%)\end{array}$ & $17(20.5)$ & $41(42.3)$ & $33(37.1)$ & 0.006 & 0.002 & 0.471 \\
\hline Antifungal therapy within 30 days before admission, $\mathrm{n}(\%)$ & $25(30.1)$ & $14(14.4)$ & $12(13.5)$ & 0.008 & 0.002 & 0.852 \\
\hline Hospitalization $\geq 48$ hours in preceding 90 days, $\mathrm{n}(\%)$ & $10(12.1)$ & $15(15.5)$ & $13(14.6)$ & 0.796 & 0.513 & 0.871 \\
\hline
\end{tabular}

NCG, non-Candida albicans colonization group; CTG, Candida albicans colonization with antifungal treatment group; CNTG, Candida albicans colonization without antifungal treatment group; HABP, Hospital-acquired bacterial pneumonia; SLE, Systemic lupus erythematosus. ${ }^{a}$, overall $\mathrm{P}$ is for comparison among no Candida albicans airway colonization, Candida albicans airway colonization with antifungal treatment and Candida albicans airway colonization without antifungal treatment group; ${ }^{\mathrm{b}}, \mathrm{P}$ is for comparison between Candida albicans airway colonization and no Candida albicans airway colonization groups; ', $\mathrm{P}$ is for comparison between Candida albicans airway colonization with or without antifungal treatment.

reveal significant difference among the three groups $(\mathrm{P}>0.05)$. More patients received immunosuppressive therapy during hospitalization, received broad-spectrum antibiotic prescription within 30 days or was residence in nursing home before admission was found in $C$. albicans airway colonization group than in non-C. albicans colonization group, $\mathrm{P}<0.05$ respectively (Table 1 ).

\section{Bacteria isolated and antimicrobial susceptibility}

Seven types of bacteria were isolated from the sputum samples of SLE patients with HABP. Among them, Acinetobacter baumannii was found as the most commonly responsible organism in both C. albicans airway colonization and non-colonization groups ( $43.5 \%$ vs. $30.1 \%, \mathrm{P}=0.031$ ). Pseudomonas aeruginosa and Klebsiella pneumoniae were the other main pathogenic bacteria. No significant difference was found in the proportion of Streptococcus pneumoniae isolation between the two groups $(7.5 \%$ vs. $12.0 \%, \mathrm{P}=0.031)$ while more MDR pathogens were isolated in C. albicans airway colonization group than the non-colonization group (58.6\% vs. 36.1\%, $\mathrm{P}=0.001$ ) (Figure 2).

\section{Risk factors of C. albicans airway colonization and MDR pathogens infection in SLE patients with HABP}

According to the baseline differences between C. albicans airway colonization group and non-colonization group, five variables with $\mathrm{P}<0.05$ were incorporated into the logistic regression model. As the results of the multivariate logistic regression model revealed, immunosuppressive therapy (OR $=1.92,95 \%$ CI: 1.32-4.06), corticosteroid therapy $>30$ days before admission ( $\mathrm{OR}=2.19,95 \%$ CI: $1.28-4.82$ ) and broad-spectrum antibiotic prescription within 30 days before admission (OR $=3.54,95 \% \mathrm{CI}$ : $1.79-4.98)$ were found to be risk factors for $C$. albicans airway colonization $(\mathrm{P}<0.05)$. C. albicans airway colonization $(\mathrm{OR}=1.92,95 \%$ CI: $1.32-4.06)$ and residence in a nursing home before admission ( $\mathrm{OR}=1.88,95 \% \mathrm{CI}: 1.28-3.29)$ were revealed as risk factors of MDR pathogen infection $(\mathrm{P}<0.05)$ (Figure 3). 

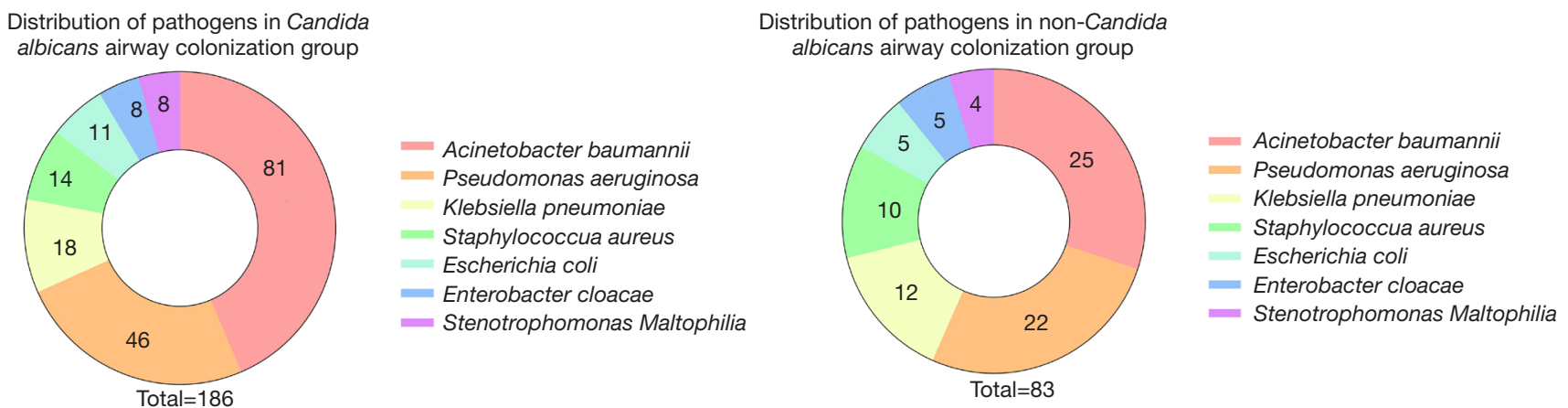

Figure 2 Distribution of bacteria caused HABP in Candida albicans airway colonization and non-colonization groups. (A) Distribution of pathogens in Candida albicans airway colonization group. Acinetobacter baumannii accounts for a larger proportion (43.5\%). (B) Distribution of pathogens in non-Candida albicans airway colonization group. The most two pathogens isolated in non-Candida albicans airway colonization group are Acinetobacter baumannii (30.1\%) and Pseudomonas aeruginosa (26.5\%).

A

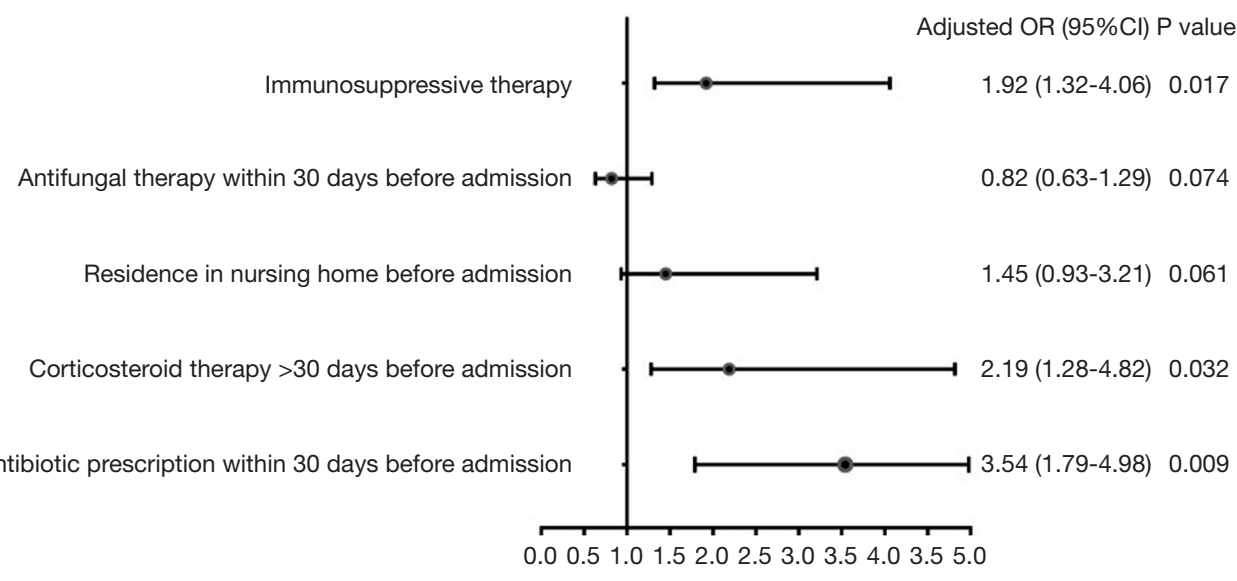

B

Broad-spectrum antibiotic prescription within 30 days before admissio

0.0 0.5 1.01 .52 .02 .53 .03 .54 .03 .55 .0

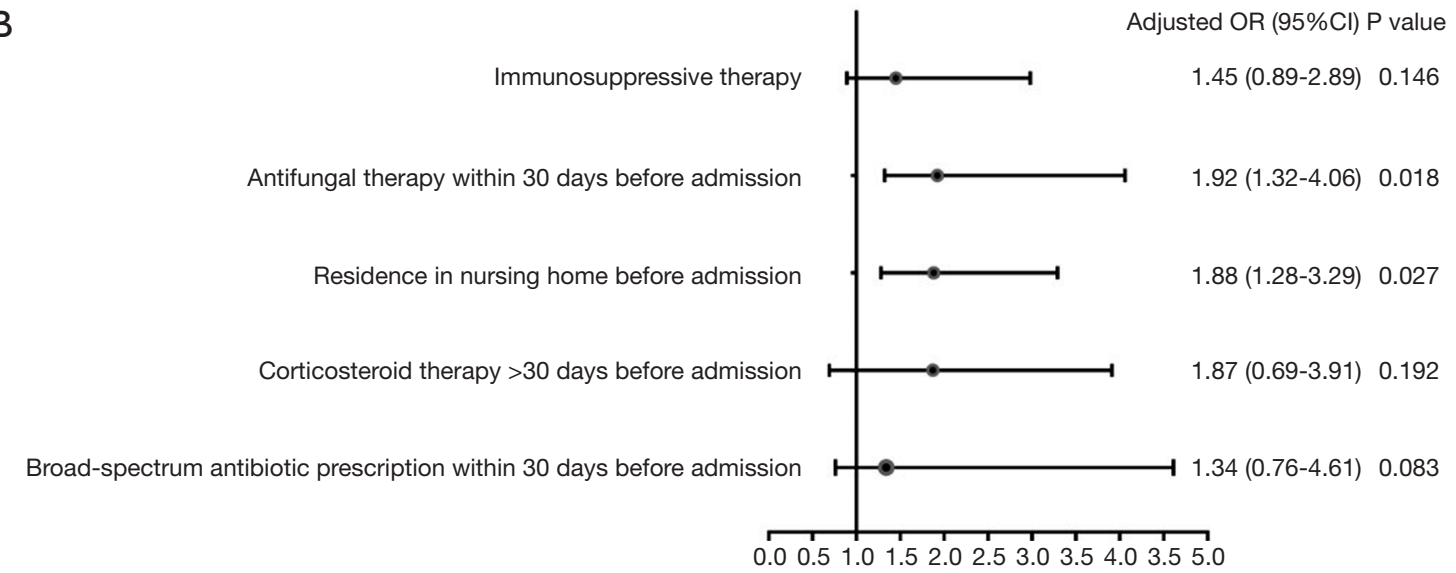

Figure 3 Risk factors of Candida albicans airway colonization and MDR pathogens infection in SLE patients with HABP. (A) Risk factors of Candida albicans airway colonization in SLE patients with HAP. (B) Risk factors of MDR pathogens infection in SLE patients with HAP. MDR, multidrug-resistant; HAP, hospital-acquired pneumonia; HABP, hospital-acquired bacterial pneumonia; SLE, systemic lupus erythematosus. 
Table 2 Duration of antibiotics treatment of different pathogens in every group

\begin{tabular}{|c|c|c|c|c|c|}
\hline Pathogens (days) & NCG & CTG & CNTG & $P$ value $^{a}$ & $P$ value ${ }^{b}$ \\
\hline MDR pathogens & $11.87 \pm 1.69$ & $12.24 \pm 2.27$ & $14.17 \pm 5.59$ & 0.014 & 0.033 \\
\hline Acinetobacter baumannii & $10.12 \pm 1.28$ & $11.21 \pm 2.45$ & $12.89 \pm 3.87$ & 0.016 & 0.025 \\
\hline Pseudomonas aeruginosa & $9.09 \pm 1.95$ & $10.57 \pm 2.66$ & $12.56 \pm 3.04$ & 0.001 & 0.024 \\
\hline Staphylococcus aureus & $9.31 \pm 1.49$ & $10.38 \pm 2.51$ & $10.33 \pm 1.51$ & 0.418 & 0.972 \\
\hline Escherichia coli & $10.02 \pm 1.23$ & $9.01 \pm 1.58$ & $10.17 \pm 1.47$ & 0.391 & 0.237 \\
\hline
\end{tabular}

NCG, non-Candida albicans colonization group; CTG, Candida albicans colonization with antifungal treatment group; CNTG, Candida albicans colonization without antifungal treatment group; MDR, multidrug-resistant. ${ }^{\text {, }}$, overall $\mathrm{P}$ is for comparison among non-Candida albicans airway colonization, Candida albicans airway colonization with antifungal treatment and Candida albicans airway colonization without antifungal treatment group; ${ }^{\mathrm{b}}, \mathrm{P}$ is for comparison between Candida albicans airway colonization with or without antifungal treatment.

\section{Duration of antibiotic therapy}

The total duration of antibiotic therapy was found significantly different among the three groups $(\mathrm{P}=0.007)$ as well as in treating MDR pathogens $(\mathrm{P}=0.014), A$. baumannii $(\mathrm{P}=0.016)$ and $P$. aeruginosa $(\mathrm{P}=0.001)$. It revealed similar length of antibiotic therapy duration in treating K. pneumoniae, $S$ aureus and Escherichia coli $(\mathrm{P}>0.05$, respectively). Antifungal treatment might decrease the duration of MDR pathogens $(\mathrm{P}=0.033)$, A baumannii $(\mathrm{P}=0.025)$ and $P$. aeruginosa $(\mathrm{P}=0.024)$ treatment in patients with $C$. albicans airway colonization (Table 2).

\section{Changes of inflammation cytokines and host immune status}

No difference was found of the six inflammation cytokines in the day of admission $\left(D_{A}\right)$ among the three groups. Concentrations of PCT and CRP on the day of HABP onset (D1) were not revealed significantly different among the three groups even in the next two weeks (D7 and D14, $\mathrm{P}>0.05$ respectively). However, a higher level of TNF- $\alpha$, INF- $\gamma$, IL-1 $\beta$, and IL-6 were found in C. albicans airway colonization group than the non-colonization group $(\mathrm{P}<0.05$ respectively). Antifungal treatment could decrease their levels in the next two weeks in CTN than in CNTG $(\mathrm{P}<0.05$ respectively) (Figure 4).

As for the immune parameters, no difference was found in the day of admission $\left(D_{A}\right)$ among the three groups as well. However, the count of CD4+Th lymphocyte and the concentration of IgA were indicated higher in the noncolonization group than in C. albicans airway colonization group $(\mathrm{P}<0.05$ respectively) while antifungal treatment could increase the levels of them in the next two weeks in CTN than in CNTG $(\mathrm{P}<0.05$ respectively). The variation trend of IL-17 was just the opposite (Figure 5).

\section{Outcome of the patients}

No significant difference of 28 days of all-cause mortality after HABP onset was found among the three groups $(10.8 \%$ vs. $13.4 \%$ vs. $15.7 \%, \mathrm{P}>0.05)$ as well as between C. albicans airway colonization group and the non-colonization group (14.5\% vs.10.8, $\mathrm{P}>0.05$ ) (Figure 6). Besides, the proportion of intensive care unit (ICU) admission and invasive mechanical ventilation application were similar $(\mathrm{P}>0.05$, respectively) (Table 3).

\section{Discussion}

Our present investigation assessed the relationship between C. albicans bronchial colonization and HABP in patients with SLE. It showed that C. albicans bronchial colonization was independently associated with MDR pathogen in SLE patients with HABP. Although it did not affect 28 days of all-cause mortality after HABP onset, it did prolong the duration of antibiotic treatment in HABP coursed by $A$. baumannii, $P$. aeruginosa, and other MDR pathogens. It was the first time that the course of antibiotic treatment was compared in HABP patients with or without airway $C$. albicans colonization which might close the evidence gap and supply experience for later researches. What is more, the 
A

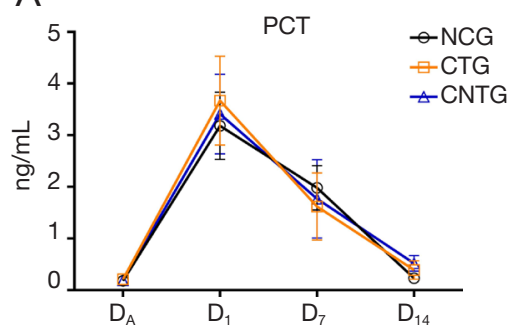

D

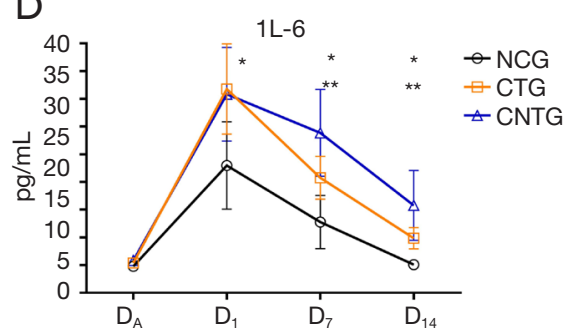

B

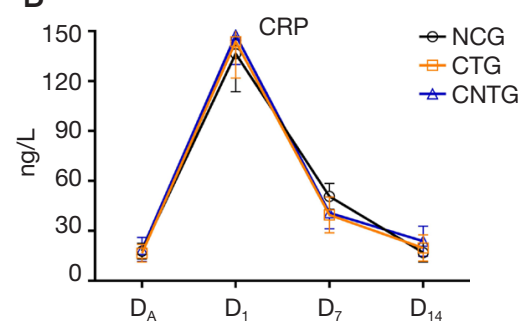

$\mathrm{E}$

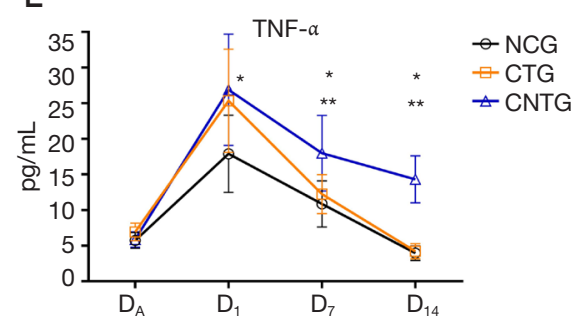

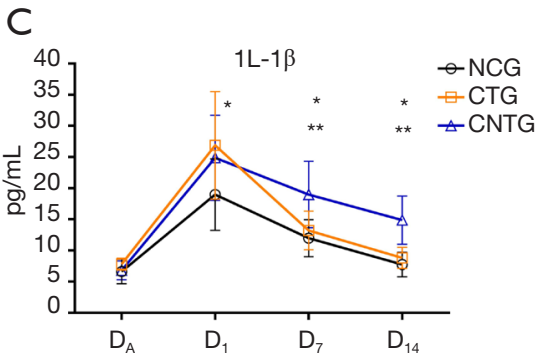

$\mathrm{F}$

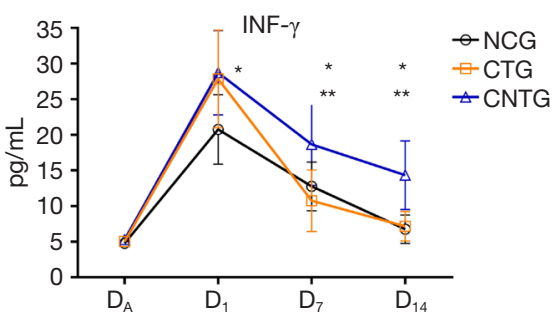

Figure 4 Evolution of inflammation cytokines during treatment of HABP in patients with SLE. (A) Evolution of PCT. (B) Evolution of CRP. (C) Evolution of IL-1ß. (D) Evolution of IL-6. (E) Evolution of TNF- $\alpha$. (F) Evolution of INF- $\gamma$. $D_{A}$ : days of admission. *, comparison among no Candida albicans airway colonization, Candida albicans airway colonization with antifungal treatment and Candida albicans airway colonization without antifungal treatment group, $\mathrm{P}<0.05 .{ }^{* *}$, comparison between Candida albicans airway colonization with or without antifungal treatment, $\mathrm{P}<0.05$. HABP, hospital-acquired bacterial pneumonia; SLE, systemic lupus erythematosus.

A
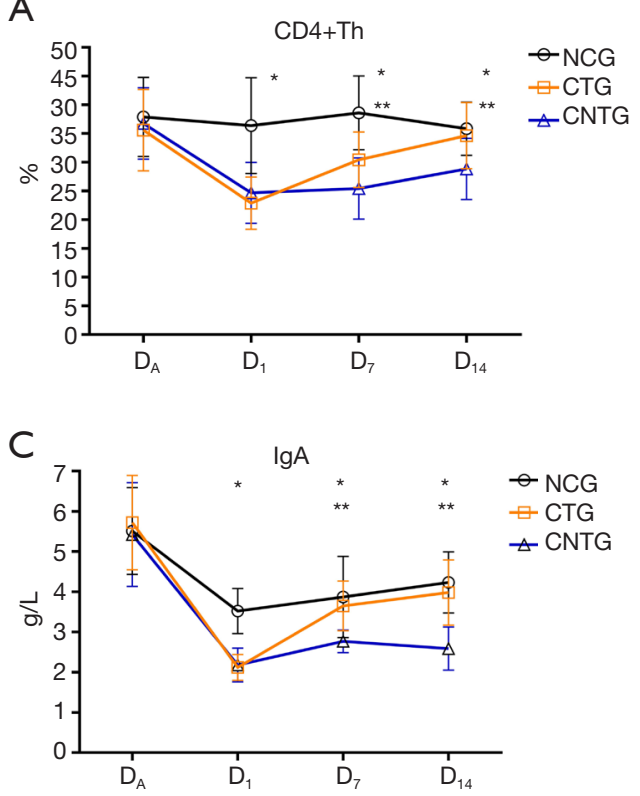

B
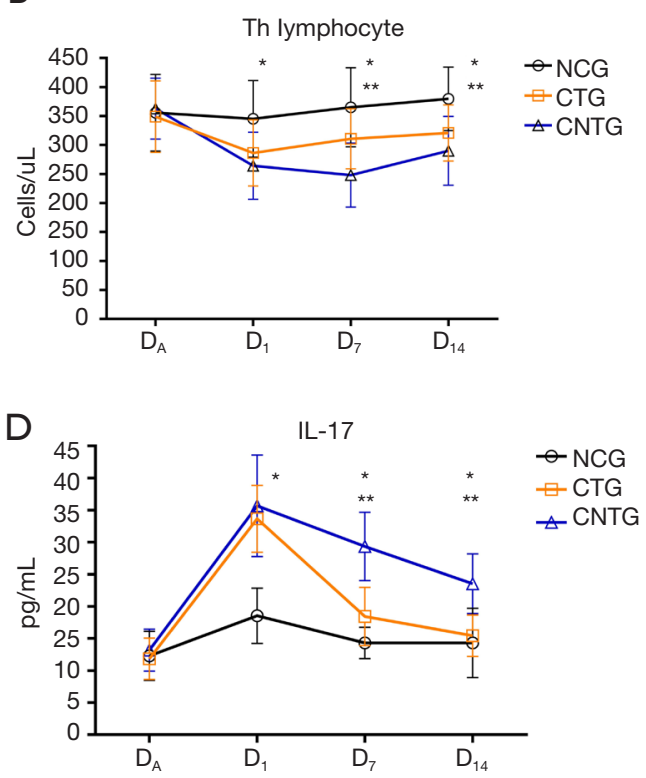

Figure 5 Evolution of immunity indexes during treatment of HABP in patients with SLE. (A) Evolution of CD4+Th lymphocyte. (B) Evolution of Th lymphocyte. (C) Evolution of IgA. (D) Evolution of IL-17. $\mathrm{D}_{\mathrm{A}}$ : days of admission. *, comparison among no Candida albicans airway colonization, Candida albicans airway colonization with antifungal treatment and Candida albicans airway colonization without antifungal treatment group, $\mathrm{P}<0.05 .{ }^{* *}$, comparison between Candida albicans airway colonization with or without antifungal treatment, $\mathrm{P}<0.05$. HABP, hospital-acquired bacterial pneumonia; SLE, systemic lupus erythematosus. 

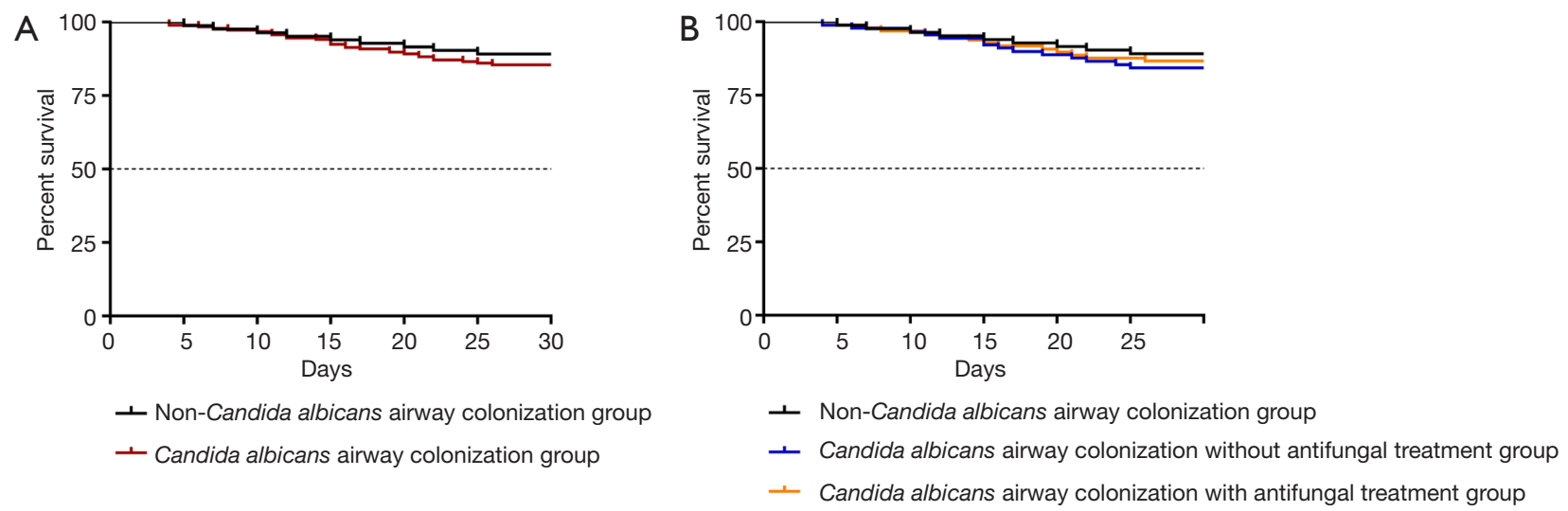

Figure 6 Twenty-eight days of all-cause mortality after HABP onset of SLE patients. The dashed black line refers to $50 \%$ of survival (median survival reference line). (A) The percent of survival of non-Candida albicans airway colonization and Candida albicans airway colonization groups. (B) The percent of survival among non-Candida albicans airway colonization, Candida albicans airway colonization with antifungal treatment and Candida albicans airway colonization without antifungal treatment groups. HABP, hospital-acquired bacterial pneumonia; SLE, systemic lupus erythematosus.

Table 3 Outcomes of SLE patients with HABP

\begin{tabular}{|c|c|c|c|c|c|}
\hline Characteristics & $\operatorname{NCG}(n=83)$ & CTG $(n=97)$ & CNTG $(n=89)$ & $P$ value ${ }^{a}$ & $P$ value \\
\hline Non-invasive mechanical ventilation, $\mathrm{n}(\%)$ & $12(14.5)$ & $16(16.5)$ & $14(15.7)$ & 0.931 & 0.954 \\
\hline Invasive mechanical ventilation, $\mathrm{n}(\%)$ & $6(7.2)$ & $6(6.2)$ & $5(5.6)$ & 0.908 & 0.883 \\
\hline 28day mortality, n (\%) & $9(10.8)$ & $13(13.4)$ & $14(15.7)$ & 0.642 & 0.809 \\
\hline
\end{tabular}

NCG, non-Candida albicans colonization group; CTG, Candida albicans colonization with antifungal treatment group; CNTG, Candida albicans colonization without antifungal treatment group; HABP, hospital-acquired bacterial pneumonia; ICU, intensive care unit; SLE, systemic lupus erythematosus. ${ }^{a}$, overall $\mathrm{P}$ is for comparison among non-Candida albicans airway colonization, Candida albicans airway colonization with antifungal treatment and Candida albicans airway colonization without antifungal treatment group; ${ }^{\mathrm{b}}$, P is for comparison between Candida albicans airway colonization with or without antifungal treatment.

concentration of some inflammatory cytokines and immune parameters were affected by the bacterial and fungal symbiosis which showed the need for further concern.

Despite a high incidence of airway Candida spp. colonization is observed in ICHs, Candida pneumonia is still an extremely rare disease (2). Meersseman's autopsy study has demonstrated that among the patients with pneumonia and Candida spp. airway colonization (77 cases), none of them was proved Candida pneumonia (15). However, Candida spp. colonization is no longer a bystander due to biofilm formation and its interplay with bacteria. What is more, whether Candida spp. airway colonization should be treated still a matter of fierce debate $(16,17)$.

We report here that $C$. albicans airway colonization could promote MDR pathogen selection. In vitro studies, it has been showed that $C$. albicans forms biofilms easily on the surface of the airway or medical devices and these biofilms play a key role in antibiotic resistance. A substantial number of bacteria can form polymicrobial biofilms with C. albicans, and these biofilm embedded pathogens are likely to acquire one or more resistant phenotypes and develop antibioticresistant patterns in this mixed culture environment $(18,19)$.

Perez's study has proved that the mucosal barrier is destroyed if the airway has been previously exposed to Candida spp. Thus, defences against $P$. aeruginosa are weakened while $C$. albicans impedes alveolar macrophage from producing reactive oxygen species (20). This phenomenon has also been confirmed in A. baumannii by Damien (4) which supplies a plausible of explanation that a higher proportion of $A$. baumannii and $P$. aeruginosa were 
isolated in C. albicans airway colonization group in our study.

Clearance of pathogens is one of the key factors that determine the course of antibiotic treatment. As is known, biofilm formation allows bacteria to escape from antimicrobial and host immunity agents as well. In Miquel's in vitro study, a panel of various bacterial species were tested in the environment of C. albicans biofilms, and hyphal binding phenomenon was confirmed by confocal and electron microscopy (21). Among them, A. baumannii and $P$. aeruginosa were two pathogens that had a high association with hyphal. Just as a finely structured building, C. albicans serves as the underlying scaffolding and bacteria forms microcolonies on the surface of biofilm. Therefore, a longer duration of antibiotic treatment was found in those with C. albicans airway colonization than in NCG, especially in the treatment of $A$. baumannii and $P$. aeruginosa pneumonia in our study. Biofilms can be eliminated by antifungal treatment, and fungi-bacteria interaction is ended which shortens the duration of antibiotic therapy.

Although Candida spp. airway colonization seldom develops to Candida pneumonia, local inflammatory response does exist. Beta-glucan is one of the components in yeast cell wall which acts as a lung proinflammatory agent causing alveolar macrophage dysfunction (22). Furthermore, it could promote the production of lung inflammatory cytokines like TNF- $\alpha$, INF- $\gamma$, IL-1 $\beta$, and IL-6, while the systemic inflammatory response cytokines like CRP and PCT are not significantly affected (23). The variation tendency could be broken by antifungal therapy which was proved in our study, and it may have a good prospect.

Biofilm formation can result in local immunity impairment. C. albicans drives the systemic expansion of fungal-specific Th17 CD4+ T cells and IL-17 responsiveness by circulating neutrophils, which synergistically protect against $C$. albicans invasive infection (24). Moreover, systemic Th17 inflammation driven by CD4+ T cells responsive to tonic stimulation by commensal $C$. albicans improves host defence against extracellular pathogens, but with potentially harmful immunological consequences (25). Thus, it was revealed in our study that the concentration of IL-17 in CNTG was the highest among the three groups while the counts of CD4+Th cells was the lowest. Antifungal treatment did change the immune function, and early recovery of immune function could prevent superinfection.

Our study proved that $C$. albicans airway colonization did not worsen the outcome of SLE patients with HABP. Neither the 28-day all-cause mortality nor the percentage of invasive mechanical ventilation was significantly affected regardless of pathogens. Furthermore, antifungal treatment also did not improve the outcome of SLE patients with $C$. albicans colonization which coincides with the earlier studies $(7,8)$. It might show that less is more in treating critically ill patients. However, antifungal therapy did shorten the course of antibiotic treatment in some pathogens, and the antibiotic-related side effects could be minimized. Anyway, the long-term effect of antifungal therapy in patients with C. albicans airway colonization is another uncertainty that highlights the need for precision medicine.

We were concerned about SLE patients with HABP and C. albicans airway colonization, with the aim of supplying a reference basis for the assessment and treatment of the disease in the future. However, limitations still exist in the research. First, C. albicans is one of the most important species of Candida spp. and NAC species isolated from sputum samples were excluded in our study due to the limited sample size. Thus, only the interactions between C. albicans and distinct species of bacteria were analyzed. Whether the mechanisms of action are the same with NAC species needs further study. Second, the population of patients enrolled was low in our study, which limited the establishment of subgroups such as the daily dose corticosteroid therapy and different drugs prescribed in immunosuppressive therapy. A growing trend of mortality was indicated in the $C$. albicans colonization group than the non-colonization group although there was no statistical significance. This may be due to the small sample size and low statistical power of this study. Further multi-center study with large sample size is needed in the future.

\section{Conclusions}

C. albicans bronchial colonization in patients with SLE did not affect the mortality of HABP, although it could prolong the duration of antibiotic treatment in HABP coursed by A. baumannii, $P$. aeruginosa and other MDR pathogens and changed the concentration of some inflammation cytokines and immune parameters.

\section{Acknowledgments}

Funding: This work was supported by the National Key Research and Development Program of China (2017YFC0909002), the Scientific Research Project of Shanghai Municipal Health Bureau (201840006) and the National Natural Science Foundation of China (81102267). 


\section{Footnote}

Conflicts of Interest: The authors have no conflicts of interests to declare.

Ethical Statement: The authors are accountable for all aspects of the work in ensuring that questions related to the accuracy or integrity of any part of the work are appropriately investigated and resolved. Ethics Committee was approved by Shanghai Jiao Tong University (No. 2016-Clinical-Res-011).

\section{References}

1. de Araújo Navas EA, Sato EI, Pereira DF, et al. Oral microbial colonization in patients with systemic lupus erythematous: correlation with treatment and disease activity. Lupus 2012;21:969-77.

2. Pappas PG, Kauffman CA, Andes DR, et al. Clinical Practice Guideline for the Management of Candidiasis: 2016 Update by the Infectious Diseases Society of America. Clin Infect Dis 2016;62:e1-50.

3. Morales DK, Hogan DA. Candida albicans interactions with bacteria in the context of human health and disease. PLoS Pathog 2010;6:e1000886.

4. Roux D, Gaudry S, Khoy-Ear L, et al. Airway fungal colonization compromises the immune system allowing bacterial pneumonia to prevail. Crit Care Med 2013;41:e191-9.

5. Tan X, Zhu S, Yan D, et al. Candida spp. airway colonization: A potential risk factor for Acinetobacter baumannii ventilator-associated pneumonia. Med Mycol 2016;54:557-66.

6. Azoulay E, Timsit JF, Tafflet M, et al. Candida colonization of the respiratory tract and subsequent pseudomonas ventilator-associated pneumonia. Chest 2006;129:110-7.

7. Timsit JF, Schwebel C, Styfalova L, et al. Impact of bronchial colonization with Candida spp. on the risk of bacterial ventilator-associated pneumonia in the ICU: the FUNGIBACT prospective cohort study. Intensive Care Med 2019;45:834-43.

8. Timsit JF, Azoulay E, Schwebel C, et al. Empirical Micafungin Treatment and Survival Without Invasive Fungal Infection in Adults With ICU-Acquired Sepsis, Candida Colonization, and Multiple Organ Failure: The EMPIRICUS Randomized Clinical Trial. JAMA 2016;316:1555-64.
9. De Pascale G, Antonelli M. Candida colonization of respiratory tract: to treat or not to treat, will we ever get an answer? Intensive Care Med 2014;40:1381-4.

10. Gordon C, Amissah-Arthur MB, Gayed M, et al. The British Society for Rheumatology guideline for the management of systemic lupus erythematosus in adults. Rheumatology 2018;57:e1-45.

11. Kalil AC, Metersky ML, Klompas M, et al. Management of Adults With Hospital-acquired and Ventilator-associated Pneumonia: 2016 Clinical Practice Guidelines by the Infectious Diseases Society of America and the American Thoracic Society. Clin Infect Dis 2016;63:e61-111.

12. Magiorakos AP, Srinivasan A, Carey RB, et al. Multidrugresistant, extensively drug-resistant and pandrug-resistant bacteria: an international expert proposal for interim standard definitions for acquired resistance. Clin Microbiol Infect 2012;18:268-81.

13. Eccles S, Pincus C, Higgins B, et al. Diagnosis and management of community and hospital acquired pneumonia in adults: summary of NICE guidance. Bmj 2014;349:g6722.

14. Aliberti S, Peyrani P, Filardo G, et al. Association between time to clinical stability and outcomes after discharge in hospitalized patients with community-acquired pneumonia. Chest 2011;140:482-8.

15. Meersseman W, Lagrou K, Spriet I, et al. Significance of the isolation of Candida species from airway samples in critically ill patients: a prospective, autopsy study. Intensive Care Med 2009;35:1526-31.

16. Stjärne Aspelund A, Hammarstrom $H$, Inghammar $M$, et al. Microbiological findings in bronchoalveolar lavage fluid from lung transplant patients in Sweden. Transpl Infect Dis 2018;20:e12973.

17. Singh A, Ralhan A, Schwarz C, et al. Fungal Pathogens in CF Airways: Leave or Treat? Mycopathologia 2018;183:119-37.

18. Hall CW, Mah TF. Molecular mechanisms of biofilmbased antibiotic resistance and tolerance in pathogenic bacteria. FEMS Microbiol Rev 2017;41:276-301.

19. Koo H, Allan RN, Howlin RP, et al. Targeting microbial biofilms: current and prospective therapeutic strategies. Nat Rev Microbiol 2017;15:740-55.

20. Pérez-Rodríguez G, Dias S, Perez-Perez M, et al. Agentbased model of diffusion of $\mathrm{N}$-acyl homoserine lactones in a multicellular environment of Pseudomonas aeruginosa and Candida albicans. Biofouling 2018;34:335-45.

21. Miquel Guennoc C, Rose C, Guinnet F, et al. A New Method for Qualitative Multi-scale Analysis of Bacterial 
Biofilms on Filamentous Fungal Colonies Using Confocal and Electron Microscopy. J Vis Exp 2017;119:54771.

22. Treviño-Rangel RJ, Gonzalez GM, Martinez-Castilla $\mathrm{AM}$, et al. Candida parapsilosis complex induces local inflammatory cytokines in immunocompetent mice. Med Mycol 2015;53:612-21.

23. Zielinski CE, Mele F, Aschenbrenner D, et al.

Pathogen-induced human TH17 cells produce IFNgamma or IL-10 and are regulated by IL-1beta. Nature
2012;484:514-8.

24. Wang L, Zhao P, Shi C, et al. Decreased levels of Th17 cells are associated with invasion fungal infections after allogeneic hematopoietic stem cell transplantation. Hematology 2018;23:148-53.

25. Shao TY, Ang WXG, Jiang TT, et al. Commensal Candida albicans Positively Calibrates Systemic Th17 Immunological Responses. Cell Host Microbe 2019;25:404-417.e6.

Cite this article as: $\mathrm{Yu}$ Y, Li J, Wang S, Gao Y, Shen H, Lu L. Effect of Candida albicans bronchial colonization on hospitalacquired bacterial pneumonia in patients with systemic lupus erythematosus. Ann Transl Med 2019;7(22):673. doi: 10.21037/ atm.2019.10.44 